\title{
T1 Erector Spinae Plane Block Catheter As a Novel Treatment Modality for Pancoast Tumor Pain
}

\author{
Hari K. Kalagara ${ }^{1}$, Paige Deichmann ${ }^{1}$, Brandon Brooks ${ }^{1}$, Peter Nagi ${ }^{1}$, Promil Kukreja ${ }^{2}$ \\ 1. Anesthesiology and Perioperative Medicine, University of Alabama, Birmingham, USA 2. Anesthesiology, University \\ of Alabama, Birmingham, USA
}

Corresponding author: Promil Kukreja, pkukreja@uabmc.edu

\begin{abstract}
Pancoast tumors are non-small cell lung tumors, which can invade the ribs, vertebrae, sympathetic ganglia and brachial plexus. In this study, a patient with right-sided Pancoast tumor presented with intractable chronic pain on the right neck, upper extremity and chest wall. The chronic pain associated with Pancoast tumor, which was difficult to treat with opioids and other medications, was effectively treated with a highthoracic erector spinae plane block (ESPB). Prolonged analgesia was provided with an ESP catheter to wean the patient from opioids. This case report provides an example where the novel interfacial ESP block can provide pain relief in challenging situations such as lung malignancies involving deeper structures and extensive areas of pain.
\end{abstract}

Categories: Anesthesiology, Pain Management, Orthopedics

Keywords: erector spinae plane block, opioid consumption, neuropathic pain, cancer pain, pain management, analgesia

Received 10/24/2019

Review began 10/29/2019 Review ended 11/05/2019 Published 11/07/2019

() Copyright 2019 Kalagara et al. This is an open access article distributed under the terms of the Creative Commons Attribution License CC-BY 3.0., which permits unrestricted use, distribution, and reproduction in any medium, provided the original author and source are credited.

\section{Introduction}

The erector spinae plane block (ESPB) is an interfascial plane block where local anesthetic (LA) is injected between the erector spine muscle and the transverse process. In 1922, Gaston Labat described similar approaches to block spinal nerves in relation to the transverse process at cervical, thoracic and lumbar vertebral levels. The ESPB was initially described to relieve chronic pain from metastatic disease and nonunion of rib fractures [1]. The ESPB has been shown to be effective in improving perioperative pain control for thoracic procedures, mastectomies, minimally invasive mitral valve and aortic valve surgery and video-assisted thoracic surgery (VATS) [2-10]. ESPB is also effective in improving postoperative pain control for abdominal, hip and pelvic surgeries, including caesarean section [11-17].

While the majority of case reports display the effectiveness of ESPB for perioperative analgesia, we present a unique case report of the successful use of ESPB for treating chronic pain due to Pancoast tumor. Initial cadaver studies revealed spread to the dorsal and ventral spinal nerve roots, and radiocontrast injection in patients confirms the spread of the injectate, resulting from the thoracic ESPB injection, into the thoracic paravertebral space across more than five intervertebral levels $[1,18]$. This extensive craniocaudal spread and analgesia from ESPB to more dermatomes from previous case reports enabled us to utilize this block in this patient to treat the severe pain affecting his right neck, shoulder, arm and chest wall.

\section{Case Presentation}

Written informed consent was provided by the patient for the procedure. A 55-year-old male of weight $66 \mathrm{~kg}$ and height $178 \mathrm{~cm}$ (body mass index [BMI], $20.8 \mathrm{~kg} / \mathrm{m}^{2}$ ), with stage IV non-small cell lung cancer, presented to the emergency department with right upper extremity, neck, chest wall and abdominal pain, nausea, and non-bloody emesis. His lung cancer was previously treated with radiation therapy as well as chemotherapy with carboplatin, paclitaxel, and, most recently, pembrolizumab. At the time of presentation, the right apical lung mass measured $10.1 \times 5.9 \mathrm{~cm}$ (Figure 1). 


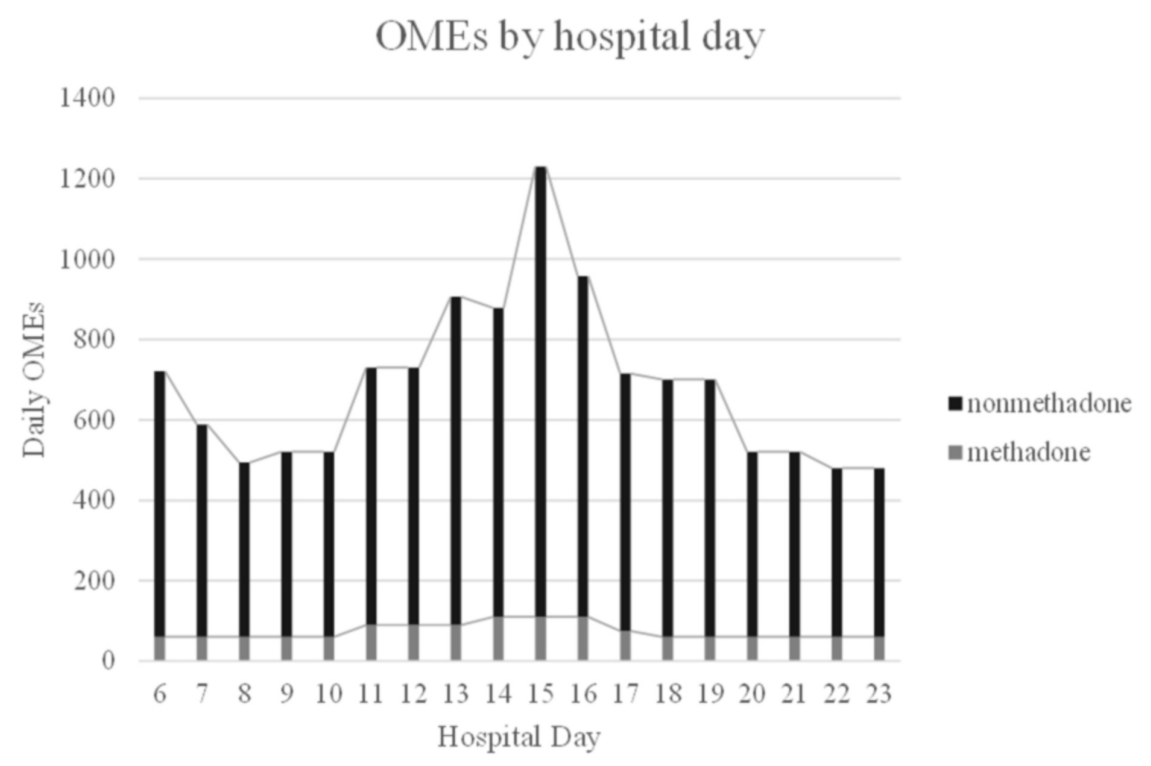

FIGURE 1: Daily OME dose of methadone and non-methadone opioids before and after ESP block catheter on day 16

OME, oral morphine equivalent; ESP, erector spinae plane

Imaging showed evidence of supraclavicular adenopathy as well as a brain metastasis to the occipital lobe, measuring $9 \times 5 \mathrm{~mm}$. The right Pancoast tumor invaded the right-sided brachial plexus and chest wall and eroded into the first and second ribs. As a result of tumor invasion, he developed chronic right upper extremity, neck and chest wall pain. His outpatient pain regimen included transdermal fentanyl $100 \mathrm{mcg}$ q72hours, oral hydromorphone $16 \mathrm{mg}$ QID PRN, and methadone $15 \mathrm{mg}$ TID. The patient underwent cervical epidural steroid injections at pain clinics in the past with transient relief.

Upon admission to the hospital, his pain regimen was transitioned from oral to intravenous agents due to his inability to tolerate oral intake in the setting of ongoing emesis. He was started on a fentanyl infusion and his home methadone regimen was converted to intravenous dosing Over the following days, his bowel regimen was medically optimized, and his nausea resolved with improved bowel function. He was transitioned back to oral opioids; however, he continued to complain of intractable pain. He described this as sharp, burning pain localized to his right neck and shoulder with radiation down to his elbow, forearm and upper anterior chest wall. He characterized the pain as $9 / 10$ in severity at baseline on a numeric rating scale (NRS) and 10/10 with activity. After a week's stay in the hospital, his daily methadone requirement had increased to $110 \mathrm{mg}$, a $244 \%$ increase from his home dose. On hospital day 16 , the pain management service was consulted for his right-sided severe pain that had not improved despite the escalation of medical therapy with opioid analgesia (Table 1 ). 


\section{Cureus}

\begin{tabular}{|c|c|c|}
\hline Hospital Day & 24 hour OME, mg (excluding methadone) & Methadone dose (mg PO) \\
\hline 6 & 661 & 60 \\
\hline 7 & 529 & 60 \\
\hline 8 & 434 & 60 \\
\hline 9 & 460 & 60 \\
\hline 10 & 460 & 60 \\
\hline 11 & 640 & 90 \\
\hline 12 & 640 & 90 \\
\hline 13 & 816 & 90 \\
\hline 14 & 768 & 110 \\
\hline 15 & 1120 & 110 \\
\hline 16 *catheter placed ${ }^{*}$ & 848 & 110 \\
\hline 17 & 640 & 75 \\
\hline 18 & 640 & 60 \\
\hline 19 & 640 & 60 \\
\hline 20 & 460 & 60 \\
\hline 21 & 460 & 60 \\
\hline 22 & 420 & 60 \\
\hline 23 & 420 & 60 \\
\hline
\end{tabular}

\section{TABLE 1: Comparison of OME opioid usage of methadone and other opioids pre and post ESP catheter placement (day 16)}

OME, oral morphine equivalent; ESP, erector spinae plane

Because of the severity of his pain, anatomically wide pain distribution and response to a prior interventional procedure (cervical epidural steroid injection), we elected to attempt a regional anesthetic technique with the hopes of weaning down his daily opioid requirements. The risks and benefits of an infraclavicular brachial plexus nerve catheter were discussed. After we reviewed prior imaging and assessed with bedside ultrasound, the infraclavicular block was ruled out due to the extent of tumor invasion and its proximity to nearby vasculature. As an alternative, we decided to proceed with an ESPB with indwelling catheter placement. Unlike a brachial plexus nerve catheter, we anticipated the ESP catheter would provide sensory blockade to not only the right upper extremity but also the proximal shoulder and right chest wall.

The ESPB was performed as follows. The patient was placed in the left lateral decubitus position and given 1 $\mathrm{mg}$ midazolam and $50 \mathrm{mcg}$ fentanyl for procedural sedation with standard monitoring. Using aseptic precautions, the skin was anesthetized with $5 \mathrm{~mL}$ of $2 \%$ lidocaine. A high-frequency linear ultrasound transducer was placed in the longitudinal parasagittal plane at the level of the T1 transverse process. A 9$\mathrm{cm}$-short bevel needle was inserted through the skin and advanced in-plane toward the T1 transverse process, and $15 \mathrm{~mL}$ of $0.5 \%$ ropivacaine was injected. We visualized the ESM separate away from the T1 transverse process and LA deposited deep into the ESP muscle. A catheter was then threaded in and left at a depth of $10 \mathrm{~cm}$ at the skin. After catheter placement was completed, the catheter was secured and then attached to an epidural catheter pump. The pump was started at a constant infusion rate of $5 \mathrm{~mL} /$ hour with $0.2 \%$ ropivacaine and a demand bolus of an additional $5 \mathrm{~mL}$ every 60 minutes. The patient reported improvement in pain immediately following the block.

On catheter day two, the pump rate was increased to allow the patient to have a demand bolus of $10 \mathrm{~mL}$ every 60 minutes while maintaining a constant rate of $5 \mathrm{~mL} /$ hour. Over the following three days, the patient experienced an improved range of motion in his right arm, which was previously limited by pain. He endorsed significant pain relief from his right chest wall to his triceps region but with some sparing of his forearm. He stated he felt "more himself in the last couple of days...the pain is out of the way enough". The 


\section{Cureus}

patient further reported that his pain had significantly diminished in severity to NRS 4/10. Simultaneously, over this time course, his daily methadone dose decreased by $54.5 \%$, from 110 to $60 \mathrm{mg}$, and daily OME of opioid usage has decreased (Figure 2).

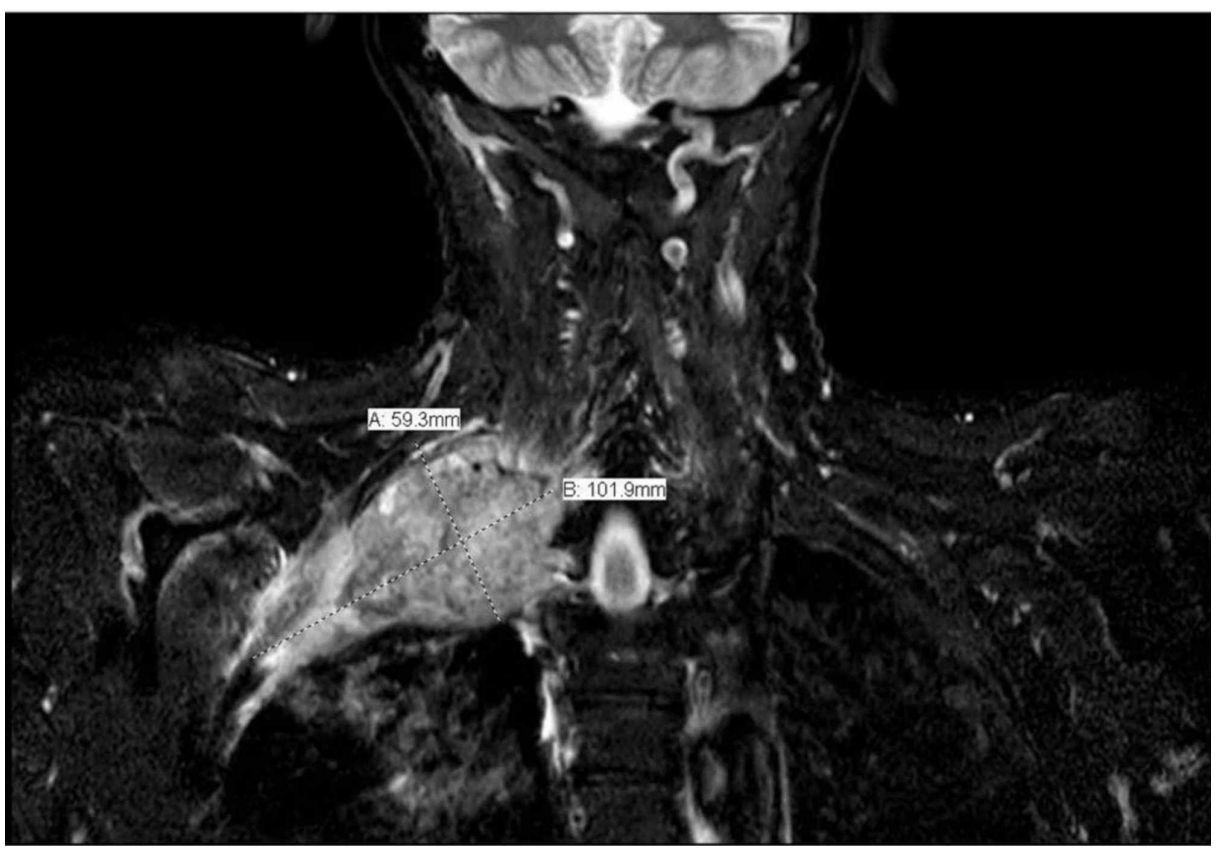

FIGURE 2: MRI of size, location and extent of right Pancoast tumor

MRI, magnetic resonance imaging

On catheter day nine, we made the decision to remove the catheter due to ongoing leukocytosis. Unfortunately, after the catheter removal, the patient pain scores increased and opioid consumption increased. The patient was followed up for the next four days as a routine follow-up after the nerve block. There was no complication or residual deficit related to ESP block after catheter removal.

\section{Discussion}

The patient in this case report presented with a wide distribution of pain in the right upper extremity, neck and anterior and lateral chest wall. The extent of Pancoast tumor and bony involvement presented as both somatic and neuropathic pain. There is a lack of robust evidence for the benefit of peripheral nerve blocks and sympathetic blocks to effectively treat neuropathic pain. There is good evidence to support the use of neuraxial blocks to control neuropathic pain, but we had some serious reservations based on the patient's clinical status and associated adverse effects with neuraxial blocks. The location of the tumor and extent of pain distribution precluded the consideration of pectoral, serratus or brachial plexus blocks. Also, these blocks would not have covered the affected area if used individually.

Because of the severity of his pain, anatomical pain distribution and good response to a prior interventional procedure (cervical epidural steroid injection), we elected to attempt a regional anesthetic technique with the hopes of weaning down his daily opioid requirements. The risks and benefits of an infraclavicular nerve catheter to provide decent analgesia were discussed. After we reviewed prior imaging and assessment with bedside ultrasound, the infraclavicular brachial plexus block was ruled out due to the extent of tumor invasion and its proximity to nearby vasculature. As an alternative, we decided to proceed with an ESPB with indwelling catheter placement.

ESPB is a relatively new regional interfacial plane block that provides somatic and visceral analgesia blocking the dorsal and ventral rami of the spinal nerve along with the rami communicantes that transmit autonomic fibers to and from the sympathetic ganglia. Dorsal ramus divides into medial and lateral cutaneous branches that innervate the skin of the back and the ventral ramus divides into lateral and anterior cutaneous branches that supply lateral and anterior chest wall. ESPB has an extensive craniocaudal spread of LA and is helpful in blocking larger dermatomes. ESPB is technically easy to perform with ultrasound guidance with fewer complications. Unlike an infraclavicular nerve catheter, we hoped the ESP catheter would provide sensory blockade not just to the right upper extremity but also to the neck and right chest wall. 
The cadaveric findings and recent case reports have indicated an optimal spread of LA when injected deep to muscle as compared to superficial to muscle. When LA is injected deep into the ESP muscle, it can reach the paravertebral space and intercostal spaces more easily and facilitates sympathetic mediated pain relief as well. The ESM extends along the length of the thoracic and lumbar spine and thus permits a craniocaudal path for LA spread. We expected that our T1 ESP block will cover lower cervical and upper thoracic spinal nerves. The patient had pain relief in most of the areas and it could not be possible with brachial plexus blocks.

The ESP block has been described for treating chronic thoracic pain, acute post-thoracotomy pain and breast surgery, as well as providing abdominal analgesia [19]. There is minimal literature describing the use of ESP block for chronic cancer pain, but it has been successfully reported for palliative pain control in a patient with pleural mesothelioma [20]. The patient in this case report did extremely well with pain control after ESP catheter placement. The patient had severe chronic pain issues with high baseline opioid use on admission. His daily opioid consumption including methadone dose was significantly reduced after ESPB catheter. The ESP catheter was functional for eight days, and patient's opioid requirements increased back to baseline after catheter removal. There are no specific guidelines for the safe and appropriate duration of ESP catheters; hence, we considered guidelines suggested for peripheral nerve blocks.

The ESP block offers many advantages, which were relevant to our patient. First, it provides coverage of multiple dermatomal levels by either a single injection or catheter. This property clearly favored ESP block over other blocks like pectoral, serratus and brachial plexus blocks.

Second, it provides a simple and safe alternative to paravertebral blocks with minimal or no risk of pneumothorax as the transverse process provides a reliable, consistent sonographic landmark, which serves as a safety backstop for needle advancement. Third, ESP block has minimal epidural spread to cause any significant sympathectomy or hypotension and more hemodynamically stable compared to neuraxial blocks.

\section{Conclusions}

This case report supports that ESP block can be used for the treatment of malignant pain in palliative care settings. This case shows the example of effective analgesia with alternative regional analgesic techniques such as ESP when there are major limitations for nerve blocks and neuraxial blocks. This novel interfascial plane can be used to treat cancer pain with an effective reduction in opioid consumption and it helps improve patient pain relief. The ESP block is one of the newer interfascial blocks with multiple potential applications, but there is limited evidence to support its efficacy. To validate the efficacy of ESP block, rigorous randomized controlled trials are warranted.

\section{Additional Information \\ Disclosures}

Human subjects: Consent was obtained by all participants in this study. University of Alabama at Birmingham (UAB) issued approval n/a. Conflicts of interest: In compliance with the ICMJE uniform disclosure form, all authors declare the following: Payment/services info: All authors have declared that no financial support was received from any organization for the submitted work. Financial relationships: All authors have declared that they have no financial relationships at present or within the previous three years with any organizations that might have an interest in the submitted work. Other relationships: All authors have declared that there are no other relationships or activities that could appear to have influenced the submitted work.

\section{References}

1. Forero M, Adhikary SD, Lopez H, Tsui C, Chin KJ: The erector spinae plane block: a novel analgesic technique in thoracic neuropathic pain. Reg Anesth Pain Med. 2016, 41:621-7. 10.1097/AAP.0000000000000451

2. Rao Kadam V, J Currie: Ultrasound-guided continuous erector spinae plane block for postoperative analgesia in video-assisted thoracotomy. Anaesth Intensive Care. 2018, 46:243-245. 10.1097/AAP.0000000000000616

3. Scimia P, Basso Ricci E, Droghetti A, Fusco P: The ultrasound-guided continuous erector spinae plane block for postoperative analgesia in video-assisted thoracoscopic lobectomy. Reg Anesth Pain Med. 2017, 42:537. 10.1097/AAP.0000000000000616

4. Singh S, Chowdhary NK: Erector spinae plane block an effective block for post-operative analgesia in modified radical mastectomy. Indian J Anaesth. 2018, 62:148-150. 10.4103/ija.IJA 72617

5. Tanaka N, Ueshima H, Otake H: Erector spinae plane block for combined lovectomy and radical mastectomys. J Clin Anesth. 2018, 45:27-28. 10.1016/j.jclinane.2017.12.012

6. Veiga M, Costa D, Brazao I: Erector spinae plane block for radical mastectomy: a new indication? . Rev Esp Anestesiol Reanim. 2018, 65:112-115. 10.1016/j.redar.2017.08.004

7. Bonvicini D, Tagliapietra L, Giacomazzi A, Pizzirani E: Bilateral ultrasound-guided erector spinae plane blocks in breast cancer and reconstruction surgery. J Clin Anesth. 2018, 44:3-4. 10.1016/j.jclinane.2017.10.006

8. Ohgoshi Y, Ikeda T, Kurahashi K: Continuous erector spinae plane block provides effective perioperative 
analgesia for breast reconstruction using tissue expanders: a report of two cases. J Clin Anesth. 2018, 44:1-2. 10.1016/j.jclinane.2017.10.007

9. Ueshima H, Hiroshi O: Transapical transcatheter aortic valve implantation performed with an erector spinae plane block. J Clin Anesth. 2018, 46:84. 10.1016/j.jclinane.2018.02.001

10. Leyva FM, Mendiola WE, Bonilla AJ, Cubillos J, Moreno DA, Chin KJ: Continuous erector spinae plane (ESP) block for postoperative analgesia after minimally invasive mitral valve surgery. J Cardiothorac Vasc Anesth. 2017, 32:2271-2274. 10.1053/j.jvca.2017.12.020

11. Restrepo-Garces, CE, Chin KJ, Suarez P, Diaz A: Bilateral continuous erector spinae plane block contributes to effective postoperative analgesia after major open abdominal surgery: a case report. A A Case Rep. 2017, 9:319-321. 10.1213/XAA.0000000000000605

12. Chin KJ, Malhas L, Perlas A: The erector spinae plane block provides visceral abdominal analgesia in bariatric surgery: a report of 3 cases. Reg Anesth Pain Med. 2017, 42:372-376.

10.1097/AAP.0000000000000581

13. Chin KJ, Adhikary S, Sarwani N, Forero M: The analgesic efficacy of pre-operative bilateral erector spinae plane (ESP) blocks in patients having ventral hernia repair. Anaesthesia. 2017, 72:452-460. 10.1111/anae.13814

14. Tulgar S, Senturk O: Ultrasound guided low thoracic erector spinae plane block for postoperative analgesia in radical retropubic prostatectomy, a new indication. J Clin Anesth. 2018, 47:4. 10.1016/j.jclinane.2018.02.013

15. Yamak Altinpulluk E, Garcia Simon D, Fajardo-Perez M: Erector spinae plane block for analgesia after lower segment caesarean section: case report. Esp Anestesiol Reanim Rev. 2018, 65:284-286.

10.1016/j.redar.2017.11.006

16. Tulgar S, Selvi O, Senturk O, Ermis MN, Ozer Z: Clinical experiences of ultrasound-guided lumbar erector spinae plane block for hip joint and proximal femur surgeries. J Clin Anesth. 2018, 47:5-6. 10.1016/j.jclinane.2018.02.014

17. Tulgar S, Senturk O: Ultrasound guided erector spinae plane block at L-4 transverse process level provides effective postoperative analgesia for total hip arthroplasty. J Clin Anesth. 2018, 44:68. 10.1016/j.jclinane.2017.11.006

18. Ueshima H, Hiroshi O: Spread of local anesthetic solution in the erector spinae plane block. J Clin Anesth. 2018, 45:23. 10.1016/j.jclinane.2017.12.007

19. Cesur S, Ay AN, Yayik AM, Naidan ME, Gurkan Y: Ultrasound-guided erector spinae plane block provides effective perioperative analgesia and anaesthesia for thoracic mass excision: a report of two cases. Anaesth Crit Care Pain Med. 2019, 38:189-190. 10.1016/j.accpm.2018.01.002

20. Ramos J, Peng P, Forero M: Long-term continuous erector spinae plane block for palliative pain control in a patient with pleural mesothelioma. Can J Anaesth. 2018, 65:852-853. 